\title{
Calculated Effect of Alloy Additions on the Saturation Magnetization of $\mathrm{Fe}_{0.80} \mathbf{B}_{0.20}$
}

D. M. C. Nicholson ${ }^{1}$, Yang Wang ${ }^{2}$, and Mike Widom ${ }^{3}$, ${ }^{1}$ Computer Science and Mathematics Division, Oak Ridge National Laboratory, Oak Ridge, TN 37830

${ }^{2}$ Pittsburgh Supercomputing Center, Pittsburgh, PA 15213

${ }^{3}$ Department of Physics, Carnegie Mellon University, Pittsburgh, PA 15213

\begin{abstract}
The great number of different local environments in amorphous alloys leads to the evolution of complicated non collinear magnetic structures. Alloy additions can affect the magnetic structure in surprising ways. For example, replacement of a small amount of $\mathrm{Fe}$ with Co increases the saturation magnetization even though Co has a much smaller moment than Fe. The calculated behavior of the magnetic structure of $\left(\mathrm{Fe}_{(\mathbf{1 - x})} \mathrm{M}_{\mathrm{x}}\right)_{\mathbf{0 . 8}} \mathrm{B}_{\mathbf{0 . 2}}$ with $\mathrm{M}=\mathrm{Co}, \mathrm{Cr}, \mathrm{Zr}$, and $\mathrm{Mn}_{2} \mathrm{Zr}$ are presented.
\end{abstract}

\section{INTRODUCTION}

For many years rapidly quenched iron based metallic glasses have been of technological importance because of their high permeability. The discovery in the past decade of alloy compositions that can form bulk metallic glasses by slow cooling from the melt has generated renewed interest in magnetic glasses. In this paper we describe our efforts to construct realistic models of the magnetic structure based on first principles calculations. For amorphous materials the importance of such models is elevated because of the difficulty of experimental structure determination. Calculations of the magnetic structure based on these structural models give values of the saturation magnetization that can be compared to measurements. These comparisons serve to corroborate the validity of the structural models.

\section{TECHNIQUE}

Our investigation begins with models of the well studied the near-eutectic glass $\mathrm{Fe}_{0.80} \mathrm{~B}_{0.20}$ to which we add $\mathrm{Co}, \mathrm{Cr}, \mathrm{Zr}$ and $\mathrm{Mn}_{2} \mathrm{Zr}$. We employ small structural models consisting of 100 atoms in a cubic cell with periodic boundary conditions. The structures are generated using the Vienna ab-initio simulation program (VASP) [1], a pseudo potential based molecular dynamics code, to obtain the liquid state. Rather than ignore local magnetic effects in the simulation of the liquid we treat the liquid as a collinear magnetic system. This treatment permits ferromagnetic and anti-ferromagnetic alignment of spin moments. This approximate inclusion of magnetic effects is found to improve the agreement between, for example, the measured and calculated pair distribution functions of liquid iron. 
VASP molecular dynamics was performed for $\mathrm{Fe}_{0.80} \mathrm{~B}_{0.20}$ to establish equilibrium at $1400 \mathrm{~K}$. The system was instantaneously quenched to the local metastable equilibrium configuration. Calculations were performed at 'medium' precision in the terminology of the VASP software; one "K-point" in the Brillouin zone was used. These choices are typical of MD for this number of atoms and give a reasonable balance between accuracy and computation time. Forces were calculated with more "K-points" for a few configurations to confirm that "K-point" convergence would not compromise the validity of the results. Similar calculations were preformed for the $\mathrm{Zr}$ and $\mathrm{Mn}_{2} \mathrm{Zr}$ containing alloys. The dilute $\mathrm{Co}$ and $\mathrm{Cr}$ compositions were obtained by direct substitution for $\mathrm{Fe}$ in the quenched $\mathrm{Fe}_{0.80} \mathrm{~B}_{0.20}$ structure. For the direct replacement of Fe the cell was bisected in the $\mathrm{x}, \mathrm{y}$, and $\mathrm{z}$, planes to form eight cubes. One Fe in each of four non adjacent small cubes was replaced at random to obtain the $4 \% \mathrm{Co}$ and $\mathrm{Cr}$ compositions. The $8 \% \mathrm{Co}$ composition was obtained by selecting one additional Fe from each of the remaining four small cubes.

The locally self consistent multiple scattering method [2], was used to determine the electronic and magnetic ground state structure within the local density approximation for the quenched structures. Angular momenta up to $1=3$ were included and all scattering from atoms within a local interaction zone of 10.0 Bohr radii was evaluated. The spherical approximation to the Kohn-Sham potential was made within the atomic spheremuffin-tin approximation [3].
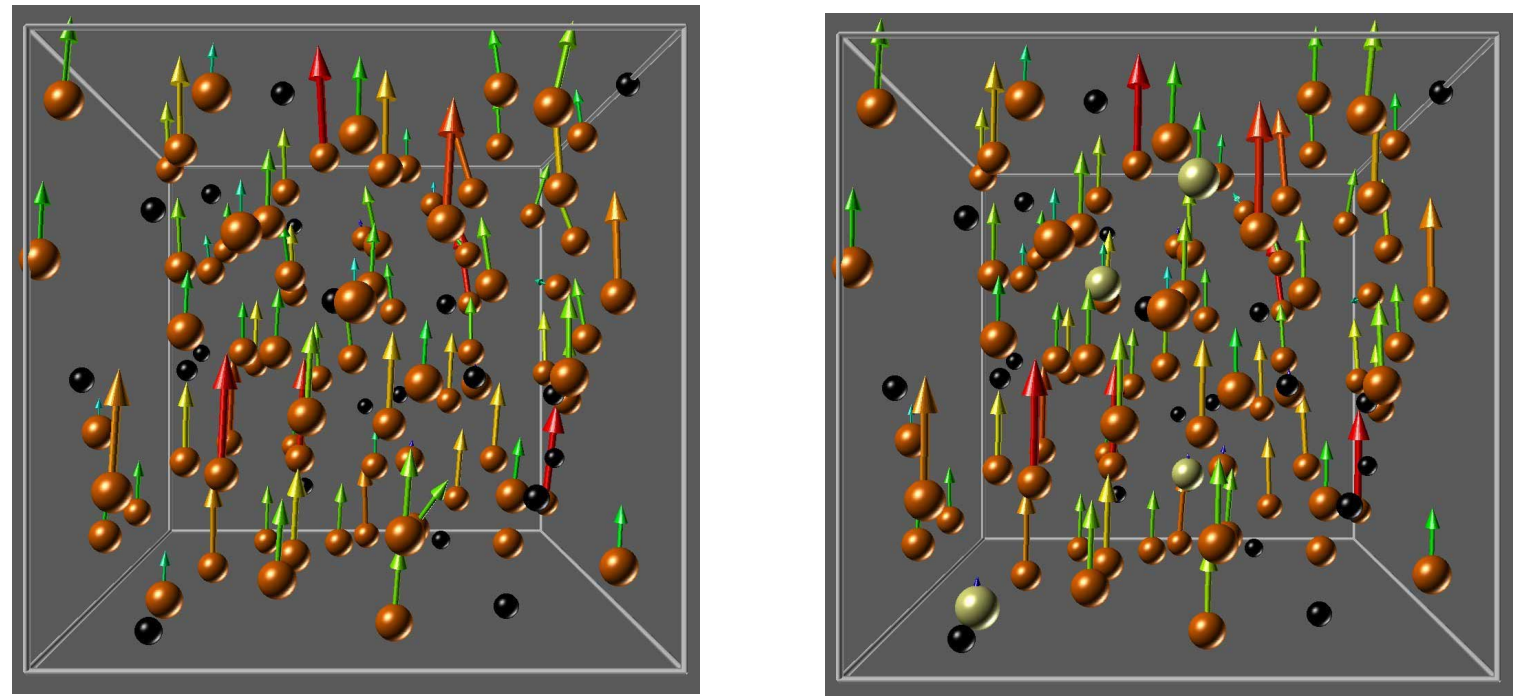

Figure 1. Moments in $\mathrm{Fe}_{0.80} \mathrm{~B}_{0.20}$ and $\mathrm{Fe}_{0.76} \mathrm{Co}_{0.04} \mathrm{~B}_{0.20}$. Red arrows indicate moments of $2.2 \mu_{\mathrm{B}}$.

\section{RESULTS}

Our starting point is the magnetic structure of $\mathrm{Fe}_{0.80} \mathrm{~B}_{0.20}$ which is compared to $\mathrm{Fe}_{0.76}$ $\mathrm{Co}_{0.04} \mathrm{~B}_{0.20}$ in Fig. 1. We find the moments to be almost collinear in $\mathrm{Fe}_{0.80} \mathrm{~B}_{0.20}$ for this calculation performed at the experimental density. There is a small amount of spread in the moment directions but no antiferromagnetically aligned Fe moments. All B atoms 
have small anti-ferromagnetic moments. For this alloy the vector sum of the moments, or saturation magnetization is $1.72 \mu_{\mathrm{B}} /$ (transition metal atom) while the sum of the local moment magnitudes is $1.85 \mu_{\mathrm{B}} /$ (transition metal atom). This agrees well with the measured magnetization $\left(1.7 \mu_{\mathrm{B}}\right.$ at $14 \% \mathrm{~B}$ and $1.84 \mu_{\mathrm{B}}$ at $\left.25 \%\right)$ [4] and with earlier calculations [5].

In Fig. 2 distributions of moment magnitudes are shown for alloys with and without $\mathrm{Zr}$. The effect of $\mathrm{Zr}$ is to broaden the distribution of moments and to reduce the average. Some atoms continue to have moments as large as in bcc Fe but on average moments are reduced considerably. Although we find no antiferromagnetic $\mathrm{Fe}$ moments for $\mathrm{Fe}_{0.80} \mathrm{~B}_{0.20}$ the addition of $\mathrm{Zr}$ results in two of the Fe moments being antiferromagnetically aligned. The moment distributions are consistent with Mossbauer measurements [6].
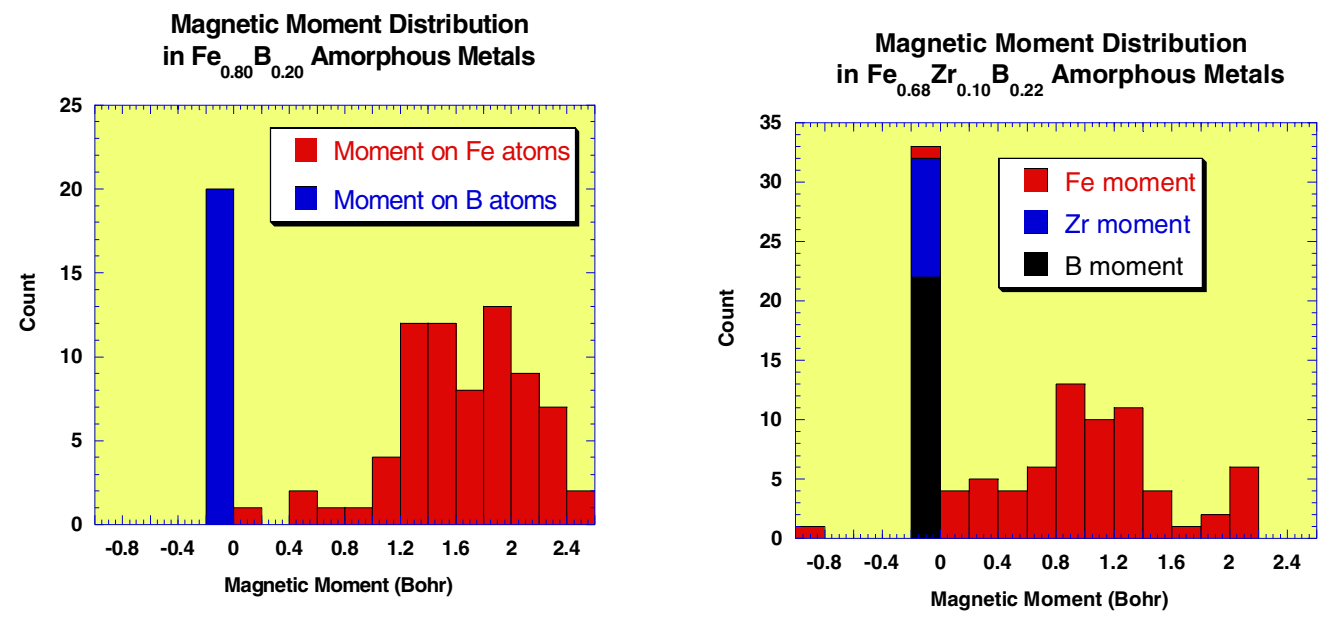

Figure 2. Distribution of local moments 


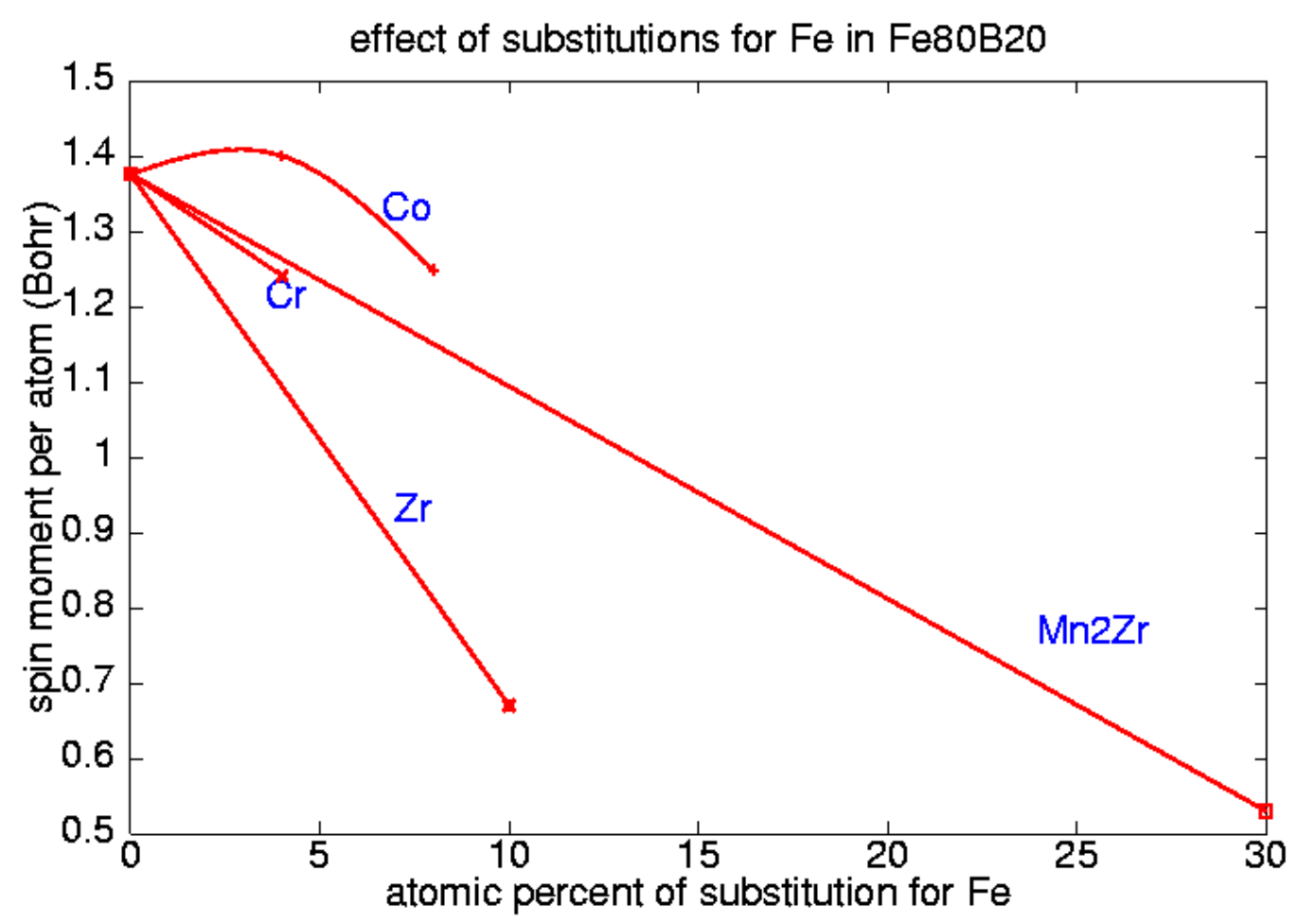

Figure 3. Saturation magnetization per atom

The impact of various additions is summarized in Fig. 3. Of the additions shown, only Co increases the saturation spin moment. One expects that as atoms with smaller moments are added the saturation magnetization would be reduced by dilution. Such a picture neglects the vector nature of the local moments. For example, although $\mathrm{Mn}$ has a large moment it has a predominantly anti-ferromagnetic exchange interaction with $\mathrm{Fe}$ and reduces the saturation magnetization by making the moments very non-collinear. This situation is depicted in Fig. 4 for $\mathrm{Fe}_{0.48} \mathrm{Mn}_{0.20} \mathrm{Zr}_{0.10} \mathrm{~B}_{0.22}$. Just the opposite is true of Co which has a very strong ferromagnetic exchange coupling with Fe. The presence of Co leads to alignment of the $\mathrm{Fe}$ moments, as seen in Fig. 1, resulting in an increased saturation magnetization of $1.75 \mu_{\mathrm{B}} /($ transition metal atom) even though the local moment magnitudes have a reduced average of $1.82 \mu_{\mathrm{B}} /$ (transition metal atom) because the Co average moment is only $1.08 \mu_{\mathrm{B}}$. The measured saturation moment peaks near 15 atomic percent Co showing a $.14 \mu_{\mathrm{B}} /$ (transition metal atom) increase due to Co [7]. Our calculated moments agree qualitatively with experiment, showing an increase of $0.03 \mu_{\mathrm{B}}$ followed by a reduction in moment by $8 \%$ Co. 


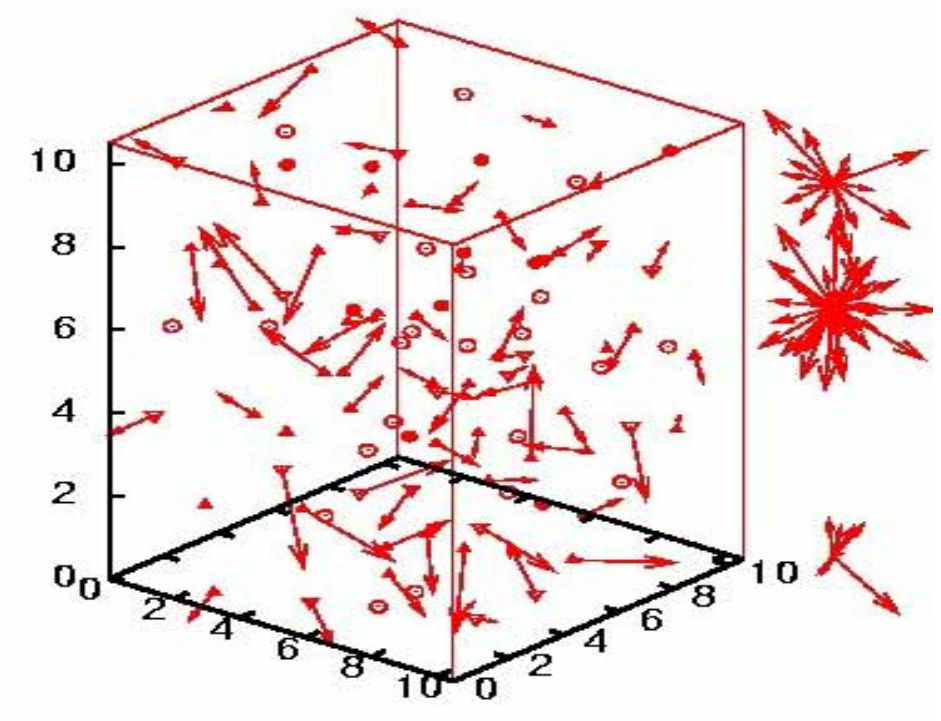

Figure 4. Moments in $\mathrm{Fe}_{0.48} \mathrm{Mn}_{0.20} \mathrm{Zr}_{0.10} \mathrm{~B}_{0.22}$. Clusters of moments represent $\mathrm{Mn}, \mathrm{Fe}$, and $\mathrm{Zr}$ ( $\mathrm{Zr}$ moments are multiplied by a factor of 10) from top to bottom.

One measure of the strength of the exchange interactions involving Co is that the energy required to reverse the moment directions of the $4 \mathrm{Co}$ atoms is $96.0 \mathrm{mRy}$ while the increase upon reversing Fe moments at these same positions is only $48.4 \mathrm{mRy}$. In an effort to obtain an estimate of the magnetic ordering energy we modeled the paramagnetic phase by randomizing the moment directions. We calculated the increase in energy within the Harris approximation upon randomizing the directions of the moments. Taking five random moment configurations for $\mathrm{Fe}_{0.80} \mathrm{~B}_{0.20}$ we obtained an energy increase of $4.2+/-0.2 \mathrm{mRy} / \mathrm{atom}$ in rough agreement with the Curie temperature, $\mathrm{k}_{\mathrm{B}} \mathrm{T}_{\mathrm{c}}=4.1 \mathrm{mRy}$ [7]. However, repeating the procedure for $\mathrm{Fe}_{.76} \mathrm{Co}_{0.04} \mathrm{~B}_{0.20}$ which has a higher Curie temperature, $\mathrm{k}_{\mathrm{B}} \mathrm{T}_{\mathrm{c}}=4.3 \mathrm{mRy}[7]$, we obtain a smaller energy difference of $3.9+/-.2 \mathrm{mRy}$ contrary to the measured increase in $\mathrm{T}_{\mathrm{c}}$ associated with Co additions. Additional calculations that do not make the Harris approximation and that go beyond our current crude approximation to the paramagnetic state will be needed to resolve this discrepancy.

\section{Conclusions}

We have performed a limited survey of the effect of substitutions on the magnetic structure of $\mathrm{Fe}_{0.80} \mathrm{~B}_{0.20}$ and found an interesting variety of effects. The most serious limitation of this study is the small size of the models. Although we can expect some self averaging over Fe environments, it is clear that for example we can claim only semiquantitative accuracy for the effect of $\mathrm{Co}$ on the magnetic structure. In spite of this limitation we find magnetic structure and mechanisms generally in agreement with observation.

Acknowledgements 
Work supported by DARPA/ONR Grant N00014-01-1-0961 and DOE Office of Basic Energy Science under subcontract DEAC05-00OR22R725464 with UT-Battelle, LLC.

\section{REFERENCES}

1. http://cms.mpi.univie.ac.at/vasp/vasp/vasp.html

2. X.-G Zhang and D.M.C. Nicholson Phys. Rev. B 60, 4551 (1999).

3. Y. Wang, G.M. Stocks, W.A. Shelton, D.M.C. Nicholson, W.M. Temmerman, and Z. Szotek, Phys. Rev. Lett. 75, 2867 (1995).

4. Ze Xianyu, Y. Ishikawa, F. Fukunaga, and N. Watanabe, J. Phys. F 15, 1799 (1975).

5. J. Hafner, M. Tegeze, and Ch. Becker, Phys. Rev B, 49285 (1994).

6. D.H. Ryan et al, Phys Rev. B 35, 8630 (1987); K.M. Unruh et al, Phys Rev. B 304968 (1084); D. Kapas et al, J Non-Cryst. Solids 156-158, 336 (1993).

7. R.C. O'Handley and D.C. Boucreaux, Phys. Status Solidi A 45, 607 (1977). Ryusuke Hasegawa, in Glassy Metals: Magnetic, Chemical, and Structural Properties, edited by Ryusuke Hasegawa (CRC Press Boca Raton, 1983) p. 165. 